\title{
Variations in apolipoprotein D and sigma non-opioid intracellular receptor 1 genes with relation to risk, severity and outcome of ischemic stroke
}

\author{
Håkan Lövkvist ${ }^{1,27^{*}}$, Ann-Cathrin Jönsson ${ }^{3}$, Holger Luthman ${ }^{4}$, Katarina Jood ${ }^{5}$, Christina Jern ${ }^{5}$, Tadeusz Wieloch ${ }^{6}$ \\ and Arne Lindgren ${ }^{1,2}$
}

\begin{abstract}
Background: In experimental studies, the apolipoprotein D (APOD) and the sigma receptor type 1 (SIGMAR1) have been related to processes of brain damage, repair and plasticity.

Methods: We examined blood samples from 3081 ischemic stroke (IS) patients and 1595 control subjects regarding 10 single nucleotide polymorphisms (SNPs) in the APOD (chromosomal location 3q29) and SIGMAR1 (chromosomal location 9p13) genes to find possible associations with IS risk, IS severity (NIHSS-score) and recovery after IS (modified Rankin Scale, mRS, at 90 days). Simple/multiple logistic regression and Spearman's rho were utilized for the analyses.

Results: Among the SNPs analyzed, rs7659 within the APOD gene showed a possible association with stroke risk $(\mathrm{OR}=1.12 ; 95 \% \mathrm{Cl}: 1.01-1.25 ; P=0.029)$ and stroke severity (NIHSS $\geq 16)(\mathrm{OR}=0.70 ; 95 \% \mathrm{Cl}: 0.54-0.92 ; P=0.009)$ when controlling for age, sex and vascular risk factors for stroke. No SNP showed an association with stroke recovery (mRS).

Conclusions: We conclude that the SNP rs7659 within the APOD gene might be related to risk and severity of ischemic stroke in patients.
\end{abstract}

Keywords: Stroke, Genetics, APOD, SIGMARI, mRS, NIHSS

\section{Background}

Stroke is a major cause of death and the main cause of adult disability. Approximately 20 to $30 \%$ of all ischemic stroke patients die in the acute stages of the stroke episode while more than one third of those who survive remain dependent of daily next-of-kin support or community care six months after stroke onset $[1,2]$. Still, five years after stroke onset, two thirds of the survivors have some neurologic impairment and disability [3]. Recanalization of occluded vessels after embolic stroke is the only therapeutic intervention available to treat acute ischemic stroke (IS), while no pharmacological treatment that stimulate brain repair or plasticity and that might enhance recovery of lost

\footnotetext{
* Correspondence: hakan.lovkvist@skane.se

'Department of Clinical Sciences Lund, Neurology, Lund University, Lund, Sweden

${ }^{2}$ Department of Neurology and Rehabilitation Medicine, Neurology, Skåne University Hospital, Lund, Sweden

Full list of author information is available at the end of the article
}

function is at hand. However, rehabilitative training such as task-oriented practice [4] and long-term progressive resistance training [5], may enhance recovery of lost brain functions.

The multifactorial and complex features of stroke impose a considerable challenge for the understanding of the pathology and for the development of new therapies. Multiple environmental factors including co-morbidities increase the risk of stroke [6]. Likewise, stroke severity is dependent on the type of stroke, density of ischemia and duration of vessel occlusion, and is also influenced by several toxic mechanisms, most identified in experimental animal models of stroke [7]. Finally, brain repair involves mechanisms differentially activated in time and space, and include inflammation, brain remodelling and relearning of activated neural networks $[8,9]$. Genetic factors influence the impact of these innate cellular mechanisms and environmental factors, affecting risk for stroke, as well as the 
severity of brain damage and the subsequent functional outcome [10].

Previous clinical studies have shown that allelic variants within the PDE4D gene, chromosome 9p21 and the ABO locus may be associated with IS risk [11-13]. Also variants in $H D A C 9$, as well as in chromosome 6p21.1 and 9p21, have shown association with large vessel IS [14-17], and variants in PITX2 and ZFXH3 may affect cardioembolic stroke risk according to other studies made $[18,19]$. In contrast to the situation regarding IS risk, reports on genetic factors contributing to outcome after stroke are scarce. However, a study has reported that the apolipoprotein $\mathrm{E}$ (APOE) $\varepsilon 2$ polymorphism might contribute to variability in outcomes after hemorrhagic stroke [20]. Likewise, an association was found between polymorphisms of the COX-2 and Glycoprotein IIIa genes on functional outcome 90-days after IS [21]. These studies clearly demonstrate the potential of genetic analysis in identifying mechanisms involved in functional recovery of stroke patients. More recently, genetic variations in the human dopamine system were associated with motor learning after stroke [22]. This indicates the potential of genetic analysis in identifying relevant mechanisms involved in stroke and therapeutic targets.

The apolipoprotein D (APOD) has been suggested to be related to stroke not only by virtue of its ability to influence trafficking of lipids but also by modulating oxidative stress, synaptic plasticity and cell death [23,24]. Moreover, APOD appears to be associated with several neurological diseases and normal ageing [25], schizophrenia [26], Alzheimer's disease (AD) $[27,28]$ and Parkinson's disease (PD) [29]. APOD levels increase with age [30], with higher levels in women than in men [25]. Also, in experimental models of stroke [31] and trauma [32], the levels of APOD are elevated. Polymorphisms of the APOD gene have been associated with increased risk of $\mathrm{AD}[28,33]$. The general increase of APOD levels in a broad range of disease states suggest that the protein may be induced in response to stress. Indeed, APOD appears to be an anti-oxidant [34] dependent on the integrity of the Met93 of this lipoprotein [35]. In animal models of stroke, increased APOD levels are correlated with better functional recovery, implying a possible function of APOD in the repair processes after stroke [31].

Whilst the apolipoproteins are trafficking lipids among cells [23], the sigma receptor type 1 (SIGMAR1, sometimes also denoted SIG1R or OPRS1) is involved in signalling and trafficking of lipids and proteins within cells [36]. Through these mechanisms the SIGMAR1 may modulate cell death and brain plasticity in experimental models of stroke [37]. The SIGMAR1 appears to play a central role in central nervous system (CNS) diseases since polymorphisms in the SIGMAR1 gene are associated with depression [38], schizophrenia [39] and alcoholism [40] as well as AD [41].
With this background we aimed to investigate whether polymorphisms in the APOD and SIGMAR1 genes influence stroke severity as well as functional outcome in patients suffering from IS. By including a group of control subjects we also assessed these polymorphisms' possible impact on IS risk.

\section{Methods}

\section{Study subjects}

The study was approved by the ethical committee at Lund University, Lund (application 543/2008). We included 2241 consecutive first-ever IS patients of all ages from Lund Stroke Register (LSR) and 840 first-ever or recurrent IS patients below 70 years of age from the Sahlgrenska Academy Study on Ischemic Stroke (SAHLSIS), Gothenburg. Both LSR and SAHLSIS have been described previously [42,43]. Patients were included if they had clinical symptoms of IS, confirmed by CT or MR or autopsy of the brain, provided DNA for analysis, and if they or their next of kin had given informed consent to participate. Exclusively for the IS risk association assessments we also included control subjects from the same geographical areas with age and gender distribution similar to those in the IS cohort. The 1595 control subjects (929 from LSR, 666 from SAHLSIS) were randomly selected from Swedish population registers from the same areas and matched for age and gender to the patients. The SAHLSIS sample included younger participants (with range 18-69 years) than the LSR sample (with range 17 to 102 years). The proportion of men was thus larger in the SAHLSIS sample (Table 1).

\section{Definition of stroke severity and stroke recovery (outcome)}

For LSR patients, initial stroke severity was assessed using the NIH stroke scale (NIHSS) in the acute phase after stroke onset [44]. For SAHLSIS patients, initial stroke severity was assessed using the Scandinavian Stroke Scale (SSS) [45]. These SSS scores were transformed to NIHSS scores through the algorithm NIHSS $=25.68-0.43 *$ SSS [46]. A NIHSS score of 8 or above but below 16 was considered to indicate a moderately severe stroke, and a score of 16 or above was considered to indicate a severe stroke [47].

For SAHLSIS, mRS at 3 months was assessed using the original scale $0-5$ at a follow-up visit with a neurologist. For LSR, stroke outcome was assessed using Riksstroke data at 3 months after stroke. We used a translation algorithm to calculate $\mathrm{mRS}$ grades from a set of self-reported functional outcome questions available in Riksstroke data [48]. The Riksstroke data do not distinguish between mRS-grades 0,1 and 2. However, as mRS-grade 2 is regarded as the upper limit for independence of help/support and the patient disability information relevant for this study is provided by mRS-grades 3,4 and 5 , we merged 
Table 1 Characteristics of control subjects and ischemic stroke (IS) cases

\begin{tabular}{|c|c|c|c|c|c|c|}
\hline & \multicolumn{2}{|l|}{ LSR } & \multicolumn{2}{|l|}{ SAHLSIS } & \multicolumn{2}{|l|}{ Combined } \\
\hline & $\begin{array}{l}\text { Controls } \\
(\mathrm{N}=929)\end{array}$ & $\begin{array}{l}\text { IS cases } \\
(\mathrm{N}=2241)\end{array}$ & $\begin{array}{l}\text { Controls } \\
(\mathrm{N}=666)\end{array}$ & $\begin{array}{l}\text { IS cases } \\
(\mathrm{N}=840)\end{array}$ & $\begin{array}{l}\text { Controls } \\
(\mathrm{N}=1595)\end{array}$ & $\begin{array}{l}\text { IS cases } \\
(\mathrm{N}=3081)\end{array}$ \\
\hline Age, Median (min, max) & $76(17,96)$ & $76(18,102)$ & $58(18,70)$ & $58(18,69)$ & $66(17,96)$ & $69(18,102)$ \\
\hline Male sex, Number (\%) & $529(57)$ & $1169(52)$ & $391(59)$ & $550(66)$ & $920(58)$ & $1719(56)$ \\
\hline Diabetes mellitus, $n$ & 925 & 2139 & 664 & 840 & 1589 & 2979 \\
\hline Number (\%) & $69(8)$ & $550(26)$ & $33(5)$ & $153(18)$ & $102(6)$ & $703(24)$ \\
\hline Hypertension, $n$ & 925 & 2183 & 665 & 829 & 1590 & 3012 \\
\hline Number (\%) & $438(47)$ & $1479(68)$ & $230(35)$ & $485(58)$ & $668(42)$ & $1964(65)$ \\
\hline Current smoking, $n$ & 927 & 2193 & 666 & 836 & 1593 & 3029 \\
\hline Number (\%) & $92(10)$ & $423(19)$ & $131(20)$ & $323(39)$ & $223(14)$ & $746(25)$ \\
\hline NIHSS at stroke onset, $n$ & - & 1983 & - & 581 & - & 2564 \\
\hline 0-7, Number (\%) & - & $1482(75)$ & - & $448(77)$ & - & $1930(75)$ \\
\hline 8-15, Number (\%) & - & $338(17)$ & - & $92(16)$ & - & $430(17)$ \\
\hline 16- , Number (\%) & - & $163(8)$ & - & $41(7)$ & - & $204(7)$ \\
\hline $\mathrm{mRS}$ at 3 months, $\mathrm{n}$ & - & 1157 & - & 565 & - & 1722 \\
\hline 0-2, Number (\%) & - & $625(54)$ & - & $435(77)$ & - & $1060(62)$ \\
\hline 3, Number (\%) & - & $196(17)$ & - & $80(14)$ & - & $276(16)$ \\
\hline 4, Number (\%) & - & $111(10)$ & - & $41(7)$ & - & $152(9)$ \\
\hline 5, Number (\%) & - & $92(8)$ & - & $2(<1)$ & - & $94(5)$ \\
\hline Deceased, Number (\%) & - & $133(12)$ & - & $7(1)$ & - & $140(8)$ \\
\hline
\end{tabular}

LSR = Lund Stroke Register, SAHLSIS = the Sahlgenska Academy Study on Ischaemic Stroke, mRS = modified Rankin Scale, NIHSS = NIH stroke scale, N = gross sample size, $n=$ net sample size after removal of missing values. All percentages are based on net sample sizes.

mRS-values 0,1 and 2 into a value of 1 [49]. In addition to the original mRS grades $0-5$, we added mRS grade 6 for individuals who had died at follow up for both samples.

\section{Phenotypes}

Definitions of intermediate phenotypes diabetes mellitus, hypertension and current smoking, and IS pathogenetic subtypes (i.e. large vessel disease, LVD; small vessel disease, SVD; and cardioembolic stroke, CE; have been described previously $[11,50,51]$.

\section{Selection of genetic variants and genotyping}

Seven SNPs in APOD and five SNPs in SIGMAR1 (or in the immediate vicinity of these regions) were selected using two different criteria: (1) SNPs serving as markers were selected based on their low pairwise linkage disequilibrium and a population frequency of $5 \%$ or more for the two gene regions $(\mathrm{N}=7)$; (2) SNPs representing non-synonymous genetic variants with low population frequency but still above $0.1 \%$ in European populations were chosen based on their probable impact on protein function $(\mathrm{N}=6)$. One of these latter non-synonymous variants, rs1800866 in SIGMAR1, is frequent enough to also be used as a marker. The genotypings were performed at our local lab in Malmö, Sweden using Sequenom technology, except for rs76929107 at the APOD locus and rs1800866 at the SIGMAR1 locus that were genotyped at LGC Genomics (former KBioscience), UK (http://www.lgcgenomics.com), using IPLEX on a MassARRAY platform (Sequenom, San Diego, CA, USA).

We scored the minor allele count of each SNP, i.e. 2, 1 or 0 , and used these in additive models. Monomorphic SNPs were excluded from further analyses.

\section{Statistical methods}

All included SNPs were tested for possible departure from Hardy-Weinberg equilibrium by chi-square test with one degree of freedom. These tests were performed on the control subjects included solely for the IS risk association analyses.

The possible association of each selected SNP with IS risk (i.e. IS patients versus control subjects) was analyzed by use of simple logistic regression, and multiple logistic regression controlling for age, gender, diabetes mellitus, hypertension and current smoking [11]. For the stroke severity response variable we used Spearman rank correlation as well as simple and logistic multiple regression with dichotomized stroke severity response (with risk category defined by NIHSS $\geq 8$ and NIHSS $\geq 16$, respectively) [47]. We also assessed functional outcome in a likewise manner (with risk category defined as $\mathrm{mRS} \geq 3$ ). 
By using non-parametric statistics for the assessments of the possible impact of polymorphisms on the NIHSS and $\mathrm{mRS}$ scores, we were able to obtain effect measures and $P$-values that were not distorted by incorrect assumptions about these non-continuous variables.

SNP rs7659 was significantly associated with stroke severity in a first-step test. We therefore performed subsequent analyses involving subgroups including study group, gender, and age $(</ \geq 70$ years) $[50,52]$. SPSS software
(PASW/SPSS, version 18, IBM Corporation, Armonk, NY, USA) was used as a computational tool for these assessments.

\section{Results}

Ischemic stroke risk

Table 2 displays (1) the frequencies of all ten nonmonomorphic SNPs for LSR and SAHLSIS joined together, and (2) the results of association analyses of these SNP

Table 2 Analysis of association between ischemic stroke risk and ten APOD and SIGMAR1 SNPs

\begin{tabular}{|c|c|c|c|c|c|}
\hline SNP* & Allele pair & $\begin{array}{l}\text { Control subj. number } \\
\text { of genotypes }\end{array}$ & $\begin{array}{l}\text { IS patients number } \\
\text { of genotypes }\end{array}$ & $\begin{array}{l}\text { Crude OR } \\
(95 \% \mathrm{CI})\end{array}$ & $\begin{array}{l}\text { Multiple LR** } \\
\text { OR }(95 \% \mathrm{Cl})\end{array}$ \\
\hline \multicolumn{6}{|l|}{ SIGMAR1: } \\
\hline \multirow[t]{3}{*}{ rs11559048 } & $\mathrm{CC}$ & 1530 & 2695 & $0.51(0.21-1.26)$ & $0.58(0.91-1.20)$ \\
\hline & $\mathrm{CT}$ & 8 & 9 & $P=0.145$ & $P=0.491$ \\
\hline & $\pi$ & 1 & - & & \\
\hline \multirow[t]{3}{*}{ rs1800866 } & $\pi$ & 1128 & 2141 & $1.03(0.92-1.16)$ & $1.02(0.90-1.16)$ \\
\hline & TG & 401 & 795 & $P=0.615$ & $P=0.768$ \\
\hline & GG & 46 & 88 & & \\
\hline \multirow[t]{3}{*}{ rs12001648 } & $\mathrm{CC}$ & 1393 & 2420 & $0.95(0.79-1.15)$ & $0.85(0.70-1.04)$ \\
\hline & CT & 165 & 286 & $P=0.595$ & $P=0.120$ \\
\hline & $\Pi$ & 9 & 8 & & \\
\hline \multirow[t]{3}{*}{ rs7036351 } & GG & 1130 & 1944 & $1.03(0.91-1.16)$ & $1.02(0.89-1.16)$ \\
\hline & GA & 399 & 693 & $P=0.675$ & $P=0.789$ \\
\hline & AA & 40 & 77 & & \\
\hline \multirow[t]{3}{*}{ rs3808873 } & GG & 833 & 1475 & $1.00(0.89-1.11)$ & $0.98(0.88-1.10)$ \\
\hline & GA & 491 & 887 & $P=0.950$ & $P=0.779$ \\
\hline & AA & 91 & 153 & & \\
\hline \multicolumn{6}{|l|}{ APOD:*** } \\
\hline \multirow[t]{3}{*}{ rs76929107 } & $\mathrm{CC}$ & 1540 & 2943 & $1.06(0.74-1.51)$ & $1.10(0.75-1.59)$ \\
\hline & CT & 44 & 91 & $P=0.761$ & $P=0.634$ \\
\hline & $\Pi$ & 1 & 1 & & \\
\hline \multirow[t]{3}{*}{ rs5952 } & $\Pi$ & 1548 & 2710 & $1.90(0.52-6.92)$ & $2.23(0.57-8.75)$ \\
\hline & TC & 3 & 10 & $P=0.329$ & $P=0.251$ \\
\hline & CC & - & - & & \\
\hline \multirow[t]{3}{*}{ rs34697430 } & GG & 435 & 769 & $1.00(0.92-1.09)$ & $1.01(0.92-1.11)$ \\
\hline & GA & 784 & 1317 & $P=0.966$ & $P=0.895$ \\
\hline & AA & 349 & 623 & & \\
\hline \multirow[t]{3}{*}{ rs7659 } & AA & 803 & 1306 & $1.11(1.01-1.22)$ & $1.12(1.01-1.25)$ \\
\hline & $A G$ & 617 & 1177 & $P=0.038$ & $P=0.029$ \\
\hline & GG & 130 & 240 & & \\
\hline \multirow[t]{3}{*}{ rs823510 } & $\pi$ & 880 & 1576 & $0.99(0.89-1.09)$ & $0.99(0.89-1.11)$ \\
\hline & TG & 590 & 981 & $P=0.805$ & $P=0.884$ \\
\hline & GG & 82 & 164 & & \\
\hline
\end{tabular}

*)All genotypes were conforming to the Hardy-Weinberg equilibrium criterion (with $P=0.093$ or more when using a chi-square test on the control subjects), except for rs11559048 that showed a significant Hardy-Weinberg disequilibrium $(P<0.001)$.

**)ORs obtained by multiple logistic regression analysis controlling for covariates age, gender, diabetes mellitus, hypertension and current smoking.

***)Two additional APOD encoding SNPs, rs5954 and rs5955, were genotyped but not included in this study due to monomorphic traits. 
frequencies against IS risk. All SNPs except rs11559048 conformed to the Hardy-Weinberg equilibrium criterion (Table 2). One SNP, rs7659 within the APOD gene region, was associated with IS risk $(\mathrm{OR}=1.11 ; 95 \% \mathrm{CI}$ : $1.01-1.22$; $P=0.038$ when tested by univariate analysis, and $\mathrm{OR}=1.12$; 95\% CI: $1.01-1.25 ; P=0.029$ when using multiple logistic regression analysis controlling for covariates age, gender, diabetes mellitus, hypertension and current smoking). However, none of these $P$-values were significant when considering Bonferroni correction for multiple testing.

\section{Stroke severity and functional outcome}

The results of the assessments of the ten nonmonomorphic SNPs of APOD and SIGMAR1 against stroke severity are presented in Table 3. Analyses using Spearman's Rho suggested that variations in one SNP, the $A P O D$-encoding rs7659, is associated with NIHSS (Rho $=-0.048 ; P=0.023$ ), while multiple logistic regression considering a NIHSS cut-off point of 16 provided an OR $=0.70 ; 95 \%$ CI: 0.54-0.92; $P=0.009$. Also, an association $(\mathrm{OR}=0.65 ; 95 \% \mathrm{CI}: 0.46-0.91 ; P=0.012)$ between the SIGMAR1 encoding rs12001648 and medium-severe stroke onset risk (NIHSS $\geq 8$ ) was found. When a subgroup of patients aged 70 years or above was tested against the severe IS onset indicator (defined as NIHSS $\geq 16$ ), an association between stroke severity and variants of SNP rs7659 within the APOD region was noticed $(\mathrm{OR}=0.63 ; 95 \% \mathrm{CI}$ : $0.45-0.88 ; P=0.006)$. These results are shown in Table 4. Still, none of these tests implied any significant association when considering Bonferroni-correction. However, when considering the pathogenetic stroke main subtype CE as a subgroup for assessment we found SNP rs7659 to be significantly associated with stroke severity defined by the NIHSS $>16$ cut point $(\mathrm{OR}=0.59 ; 95 \% \mathrm{CI}: 0.40-0.85$; $P=0.005$; results shown in Table 4 ).

None of the ten non-monomorphic SNPs significantly affected functional outcome after stroke (Table 5).

\section{Discussion}

With this large study sample comprising a total of 3081 IS patients we were able to perform analyses aimed to find possible impact of selected polymorphisms encoding for APOD and SIGMAR1 on (1) stroke severity and (2) stroke outcome, respectively. By adding 1595 control subjects not suffering any stroke onset from the same geographical areas and with the same age and gender distribution as the IS patients, we have also been able to examine possible effect of these polymorphisms on IS risk.

The conclusions from the non-parametric Spearman correlation analyses (NIHSS and mRS-scores, respectively, against SNP variations) were based upon $P$-values obtained by using a "conservative" approach providing high adequacy at the cost of some statistical power loss. The transformation of these numerical variables into dichotomized indicators (coded 1 or 0 ) also caused information loss. On the other hand this enabled us to focus on possible threshold effects when examining e.g. the genetic effect on IS severity (by using NIHSS cutpoints $0-7$ vs. 8 or above, or $0-15$ vs. 16 or above).

Table 3 Analysis of association between stroke severity (NIHSS) and ten APOD and SIGMAR1 SNPs

\begin{tabular}{|c|c|c|c|c|c|c|c|c|c|c|}
\hline \multirow[b]{3}{*}{ SNP } & \multirow{3}{*}{$\begin{array}{l}\text { NIHSS score } \\
\text { Estimated } \\
\text { Spearman's Rho }\end{array}$} & \multirow[b]{3}{*}{$P$-value } & \multicolumn{4}{|c|}{$\begin{array}{l}\text { NIHSS; dichotomous indicator of medium-severe } \\
\text { (vs. mild) ischemic stroke onset (NIHSS } \geq 8 \text { ) }\end{array}$} & \multicolumn{4}{|c|}{$\begin{array}{l}\text { NIHSS; dichotomous indicator of severe } \\
\text { (vs. mild-medium) ischemic stroke onset } \\
\text { (NIHSS } \geq 16 \text { ) }\end{array}$} \\
\hline & & & \multicolumn{2}{|l|}{$\begin{array}{l}\text { Simple logistic } \\
\text { regression }\end{array}$} & \multicolumn{2}{|c|}{$\begin{array}{l}\text { Multiple logistic } \\
\text { regression }\end{array}$} & \multicolumn{2}{|l|}{$\begin{array}{l}\text { Simple logistic } \\
\text { regression }\end{array}$} & \multicolumn{2}{|c|}{$\begin{array}{l}\text { Multiple logistic } \\
\text { regression }\end{array}$} \\
\hline & & & OR $(95 \% \mathrm{Cl})$ & $P$-value & OR (95\% CI) & $P$-value & OR $(95 \% \mathrm{Cl})$ & $P$-value & OR $(95 \% \mathrm{Cl})$ & $P$-value \\
\hline \multicolumn{11}{|l|}{ SIGMAR1: } \\
\hline rs11559048 & 0.023 & 0.284 & $1.86(0.44-7.79)$ & 0.399 & $1.74(0.40-7.69)$ & 0.462 & $\left.-{ }^{*}\right)$ & - & $\left.--^{*}\right)$ & - \\
\hline rs1800866 & -0.012 & 0.554 & $1.02(0.87-1.22)$ & 0.772 & $0.98(0.81-1.17)$ & 0.804 & $0.97(0.75-1.25)$ & 0.822 & $0.87(0.64-1.17)$ & 0.347 \\
\hline rs12001648 & -0.044 & 0.040 & $0.77(0.56-1.05)$ & 0.094 & $0.65(0.46-0.91)$ & 0.012 & $1.01(0.66-1.56)$ & 0.967 & $0.75(0.48-1.27)$ & 0.283 \\
\hline rs7036351 & 0.000 & 0.984 & $1.04(0.87-1.25)$ & 0.670 & $0.99(0.81-1.21)$ & 0.922 & $0.94(0.71-1.25)$ & 0.664 & $0.84(0.61-1.16)$ & 0.290 \\
\hline rs3808873 & 0.011 & 0.627 & $1.04(0.88-1.23)$ & 0.619 & $1.07(0.90-1.27)$ & 0.457 & $0.79(0.61-1.03)$ & 0.084 & $0.77(0.57-1.03)$ & 0.079 \\
\hline \multicolumn{11}{|l|}{ APOD: } \\
\hline rs76929107 & 0.004 & 0.826 & $0.85(0.50-1.46)$ & 0.555 & $0.74(0.40-1.36)$ & 0.327 & $0.97(0.45-2.12)$ & 0.948 & $1.17(0.50-2.73)$ & 0.722 \\
\hline rs5952 & 0.022 & 0.309 & $1.54(0.38-6.18)$ & 0.542 & $1.40(0.34-5.79)$ & 0.641 & $\left.--^{*}\right)$ & - & $\left.--^{*}\right)$ & - \\
\hline rs34697430 & 0.011 & 0.599 & $1.07(0.93-1.23)$ & 0.334 & $1.08(0.94-1.25)$ & 0.280 & $1.16(0.95-1.42)$ & 0.154 & $1.20(0.96-1.50)$ & 0.116 \\
\hline rs7659 & -0.048 & 0.023 & 0.89 (0.77-1.04) & 0.141 & $0.85(0.72-1.00)$ & 0.044 & $0.84(0.66-1.06)$ & 0.135 & $0.70(0.54-0.92)$ & 0.009 \\
\hline rs823510 & 0.013 & 0.538 & $1.03(0.88-1.21)$ & 0.680 & $1.00(0.85-1.19)$ & 0.965 & $1.06(0.83-1.34)$ & 0.658 & $0.96(0.73-1.25)$ & 0.736 \\
\hline
\end{tabular}


Table 4 Detailed assessment of possible association between stroke severity (NIHSS) and SNP rs7659 within APOD

\begin{tabular}{|c|c|c|c|c|c|c|c|c|c|c|}
\hline \multirow{3}{*}{ Subgroup } & \multirow{3}{*}{$\begin{array}{l}\text { NIHSS score } \\
\text { Estimated } \\
\text { Spearman's Rho }\end{array}$} & \multirow{3}{*}{$P$-value } & \multicolumn{4}{|c|}{$\begin{array}{l}\text { NIHSS; dichotomous indicator of medium-severe } \\
\text { (vs. mild) ischemic stroke onset (NIHSS } \geq 8 \text { ) }\end{array}$} & \multicolumn{4}{|c|}{$\begin{array}{l}\text { NIHSS; dichotomous indicator of severe } \\
\text { (vs. mild-medium) ischemic stroke onset } \\
\text { (NIHSS } \geq 16 \text { ) }\end{array}$} \\
\hline & & & \multicolumn{2}{|l|}{$\begin{array}{l}\text { Simple logistic } \\
\text { regression }\end{array}$} & \multicolumn{2}{|c|}{$\begin{array}{l}\text { Multiple logistic } \\
\text { regression }\end{array}$} & \multicolumn{2}{|l|}{$\begin{array}{l}\text { Simple logistic } \\
\text { regression }\end{array}$} & \multicolumn{2}{|c|}{$\begin{array}{l}\text { Multiple logistic } \\
\text { regression }\end{array}$} \\
\hline & & & OR $(95 \% \mathrm{Cl})$ & $P$-value & OR $(95 \% \mathrm{Cl})$ & $P$-value & OR $(95 \% \mathrm{Cl})$ & $P$-value & OR $(95 \% \mathrm{Cl})$ & $P$-value \\
\hline Total & -0.048 & 0.023 & $0.89(0.77-1.04)$ & 0.141 & $0.85(0.72-1.00)$ & 0.044 & $0.84(0.66-1.06)$ & 0.135 & $0.70(0.54-0.92)$ & 0.009 \\
\hline \multirow[t]{2}{*}{$(\mathrm{N}=2221)$} & & & 1676 controls & & 1618 controls & & 2023 controls & & 1947 controls & \\
\hline & & & 545 cases & & 495 cases & & 198 cases & & 166 cases & \\
\hline Males & -0.055 & 0.053 & $0.89(0.72-1.11)$ & 0.293 & $0.85(0.68-1.06)$ & 0.143 & $0.82(0.58-1.17)$ & 0.273 & $0.75(0.52-1.10)$ & 0.144 \\
\hline \multirow[t]{2}{*}{$(\mathrm{N}=1232)$} & & & 971 controls & & 939 controls & & 1146 controls & & 1105 controls & \\
\hline & & & 261 cases & & 243 cases & & 86 cases & & 77 cases & \\
\hline Females & -0.040 & 0.209 & $0.90(0.73-1.11)$ & 0.311 & $0.85(0.68-1.07)$ & 0.168 & $0.85(0.62-1.16)$ & 0.311 & $0.66(0.46-0.96)$ & 0.028 \\
\hline \multirow[t]{2}{*}{$(\mathrm{N}=989)$} & & & 705 controls & & 679 controls & & 877 controls & & 842 controls & \\
\hline & & & 284 cases & & 252 cases & & 112 cases & & 89 cases & \\
\hline LSR & -0.054 & 0.026 & $0.87(0.73-1.03)$ & 0.113 & $0.81(0.67-0.98)$ & 0.026 & $0.79(0.61-1.03)$ & 0.081 & $0.65(0.48-0.88)$ & 0.005 \\
\hline \multirow[t]{2}{*}{$(N=1683)$} & & & 1263 controls & & 1204 controls & & 1523 controls & & 1450 controls & \\
\hline & & & 420 cases & & 377 cases & & 160 cases & & 133 cases & \\
\hline SAHLSIS & -0.032 & 0.454 & $0.97(0.71-1.33)$ & 0.858 & $0.94(0.68-1.30)$ & 0.702 & $1.03(0.62-1.73)$ & 0.902 & $0.86(0.48-1.53)$ & 0.605 \\
\hline \multirow[t]{2}{*}{$(N=538)$} & & & 413 controls & & 412 controls & & 500 controls & & 497 controls & \\
\hline & & & 125 cases & & 118 cases & & 38 cases & & 33 cases & \\
\hline Age $<70$ & -0.066 & 0.029 & $0.89(0.70-1.13)$ & 0.333 & $0.85(0.67-1.09)$ & 0.201 & $0.98(0.66-1.47)$ & 0.940 & $0.81(0.51-1.27)$ & 0.348 \\
\hline \multirow[t]{2}{*}{$(\mathrm{N}=1084)$} & & & 868 controls & & 853 controls & & 1021 controls & & 1001 controls & \\
\hline & & & 216 cases & & 202 cases & & 63 cases & & 54 cases & \\
\hline Age $\geq 70$ & -0.042 & 0.156 & $0.88(0.72-1.08)$ & 0.215 & $0.82(0.66-1.02)$ & 0.080 & $0.76(0.57-1.01)$ & 0.060 & $0.63(0.45-0.88)$ & 0.006 \\
\hline \multirow[t]{2}{*}{$(\mathrm{N}=1137)$} & & & 808 controls & & 750 controls & & 1002 controls & & 946 controls & \\
\hline & & & 329 cases & & 266 cases & & 135 cases & & 112 cases & \\
\hline LVD & -0.008 & 0.918 & $1.14(0.70-1.87)$ & 0.597 & $0.97(0.57-1.66)$ & 0.924 & $1.20(0.54-2.67)$ & 0.651 & $0.53(0.17-1.65)$ & 0.272 \\
\hline \multirow[t]{2}{*}{$(N=185)$} & & & 133 controls & & 128 controls & & 170 controls & & 163 controls & \\
\hline & & & 52 cases & & 45 cases & & 15 cases & & 10 cases & \\
\hline SVD & -0.019 & 0.667 & $0.77(0.46-1.30)$ & 0.329 & $0.81(0.47-1.38)$ & 0.432 & N/A & & N/A & \\
\hline \multirow[t]{2}{*}{$(N=500)$} & & & 461 controls & & 452 controls & & & & & \\
\hline & & & 39 cases & & 38 cases & & & & & \\
\hline CE & -0.076 & 0.065 & $0.89(0.70-1.15)$ & 0.367 & $0.78(0.59-1.03)$ & 0.081 & $0.72(0.52-1.00)$ & 0.051 & $0.59(0.40-0.85)$ & 0.005 \\
\hline \multirow[t]{2}{*}{$(\mathrm{N}=595)$} & & & 364 controls & & 338 controls & & 484 controls & & 448 controls & \\
\hline & & & 231 cases & & 204 cases & & 111 cases & & 94 cases & \\
\hline
\end{tabular}

Ischemic stroke patients from Lund Stroke Register (LSR) and the Sahlgenska Academy Study on Ischemic Stroke (SAHLSIS).

NIHSS = NIH stroke scale. Multiple logistic regression models are controlling for covariates age, gender, diabetes mellitus, hypertension and current smoking when analyzing the entire sample as well as separate study groups and age groups. When analyzing males and females separately the covariate gender is omitted from these multivariable models. Pathogenetic ischemic stroke subtype: $L V D=$ Large vessel disease; $S V D=S m a l l$ vessel disease; $C E=C$ ardioembolic stroke. $N / A=$ not applicable due to absence of sampling units with NIHSS $\geq 16$.

The SIGMAR1 region on chromosome 9p13 displays two polymorphisms that have a strong influence in CNS disease, namely rs1799729 (GC-241-240TT) and rs1800866 (Gln2Pro) that show LD forming haplotypes GC-Q and TT-P [39-41]. The rs1799729 is found in the proximal promoter region while rs1800866 is present in the first exon. Only rs1800866 was analysed in our study but these two SNPs are closely related (nearly in complete
LD with $\mathrm{r}^{2}=0.98$ ) and have been reported to be associated with neuroprotection and risk of AD [41], and also risk of depression and alcoholism [38,40]. The polymorphism Gln2Pro is located in the amino acid sequence motif MQWAVGRR [53] at the N-terminal part of the protein, which is an endoplasmatic binding region. Hence, a mutation could affect trafficking of SIGMAR1 associated processes, which have been implicated in rodent models 
Table 5 Analysis of association between outcome after stroke (shown by modified Rankin Scale, mRS) and ten APOD and SIGMAR1 SNPs

\begin{tabular}{|c|c|c|c|c|c|c|c|c|c|c|c|c|}
\hline \multirow{4}{*}{ SNP } & \multicolumn{6}{|c|}{ Deceased patients are not included in mRS: } & \multicolumn{6}{|c|}{ Deceased patients are included in mRS: } \\
\hline & \multicolumn{2}{|l|}{ Ordinal score: } & \multicolumn{4}{|c|}{ Dichotomous indicator for dependence of support: } & \multicolumn{2}{|l|}{ Ordinal score: } & \multicolumn{4}{|c|}{ Dichotomous indicator for dependence of support: } \\
\hline & \multirow{2}{*}{$\begin{array}{l}\text { Estimated } \\
\text { Spearman's Rho }\end{array}$} & \multirow[t]{2}{*}{$P$-value } & \multicolumn{2}{|c|}{ Simple logistic regression } & \multicolumn{2}{|c|}{ Multiple logistic regression } & \multirow{2}{*}{$\begin{array}{l}\text { Estimated } \\
\text { Spearman's Rho }\end{array}$} & \multirow[t]{2}{*}{$P$-value } & \multicolumn{2}{|c|}{ Simple logistic regression } & \multicolumn{2}{|c|}{ Multiple logistic regressior } \\
\hline & & & OR $(95 \% \mathrm{Cl})$ & $P$-value & OR $(95 \% \mathrm{Cl})$ & $P$-value & & & OR $(95 \% \mathrm{Cl})$ & $P$-value & OR $(95 \% \mathrm{Cl})$ & $P$-value \\
\hline \multicolumn{13}{|l|}{ SIGMAR1: } \\
\hline rs11559048 & 0.045 & 0.081 & $3.35(0.80-14.1)$ & 0.099 & $2.75(0.49-15.3)$ & 0.249 & 0.026 & 0.286 & $2.64(0.63-11.1)$ & 0.185 & $2.29(0.38-13.7)$ & 0.364 \\
\hline rs1800866 & 0.011 & 0.655 & $1.13(0.93-1.37)$ & 0.231 & $1.10(0.88-1.36)$ & 0.404 & 0.004 & 0.872 & $1.08(0.90-1.30)$ & 0.402 & $1.06(0.85-1.31)$ & 0.612 \\
\hline rs12001648 & -0.012 & 0.631 & $0.94(0.67-1.32)$ & 0.712 & $0.77(0.53-1.11)$ & 0.165 & -0.001 & 0.963 & $0.98(0.72-1.34)$ & 0.892 & $0.75(0.53-1.07)$ & 0.115 \\
\hline rs7036351 & 0.006 & 0.817 & $1.10(0.89-1.33)$ & 0.390 & $1.03(0.83-1.29)$ & 0.781 & -0.007 & 0.772 & $1.04(0.86-1.25)$ & 0.722 & $0.97(0.79-1.21)$ & 0.810 \\
\hline rs3808873 & 0.021 & 0.436 & $1.08(0.90-1.29)$ & 0.392 & $1.11(0.91-1.35)$ & 0.290 & 0.012 & 0.636 & $1.05(0.89-1.24)$ & 0.598 & $1.07(0.89-1.30)$ & 0.457 \\
\hline \multicolumn{13}{|l|}{ APOD: } \\
\hline rs76929107 & 0.002 & 0.947 & $0.98(0.54-1.78)$ & 0.943 & $0.95(0.49-1.82)$ & 0.868 & -0.013 & 0.582 & $0.87(0.49-1.54)$ & 0.623 & $0.92(0.48-1.73)$ & 0.790 \\
\hline rs5952 & 0.016 & 0.520 & $1.98(0.28-14.1)$ & 0.494 & $2.90(0.32-26.7)$ & 0.346 & 0.025 & 0.306 & $2.35(0.39-14.1)$ & 0.350 & $3.09(0.35-27.5)$ & 0.312 \\
\hline rs34697430 & -0.019 & 0.460 & $0.91(0.78-1.06)$ & 0.221 & $0.91(0.77-1.07)$ & 0.243 & 0.002 & 0.946 & $0.96(0.83-1.10)$ & 0.514 & $0.92(0.79-1.08)$ & 0.295 \\
\hline rs7659 & 0.002 & 0.929 & $1.03(0.88-1.22)$ & 0.684 & $0.98(0.82-1.17)$ & 0.832 & -0.005 & 0.846 & $1.02(0.87-1.18)$ & 0.842 & $0.96(0.81-1.14)$ & 0.665 \\
\hline rs823510 & 0.012 & 0.653 & $1.01(0.85-1.21)$ & 0.882 & $1.02(0.84-1.23)$ & 0.871 & -0.008 & 0.742 & $0.97(0.82-1.14)$ & 0.696 & $0.97(0.81-1.17)$ & 0.764 \\
\hline
\end{tabular}

Ischemic stroke patients from Lund Stroke Register and the Sahlgenska Academy Study on Ischemic Stroke.

$\mathrm{mRS}=$ modified Rankin Scale. Ordinal score comprises distinguishable categories $0-2,3,4,5$ and, if deceased patients are included, also category 6. Correspondingly, dichotomous indicator comprises categories $0-2$ as control subjects and category 3 or above as cases. 
of stroke [37]. However; we could not find any association between the SIGMAR1 polymorphisms and stroke risk, severity or recovery. The significance of the weak association of rs12001648 needs further investigation.

Although rigorous statistical analysis did not provide clear evidence of an association between the APOD SNPs and stroke risk, severity or outcome, the possible genetic influence of polymorphism rs7659 is interesting and potentially relevant. Rs7659 is located in the 3'UTR of $A P O D$, and a functional variant in this area might influence the transcription of the gene or mRNA splicing. Indeed, this SNP appears to be positioned at a putative binding site for the human splicing factor SR SC35 [54]. Also, it is previously shown that rs7659 may be associated with early onset $\mathrm{AD}$ within the subgroup of patients lacking the APOE\&4 allele [28] and with long term clinical outcome in schizophrenics [54]. Moreover, the $A P O D$ gene is localized on chromosome $3 \mathrm{q} 2.2$-qter in close proximity to the $3 \mathrm{q} 25-26$ region linked to $A D$ [24]. Hence, taking into consideration the association between rs7659 to other CNS disease and our finding of a possible association of rs7659 with stroke risk and stroke severity, particularly among the elderly, this strongly encourages further studies of rs7659.

Possible occurrence of false positive $P$-values was supressed by Bonferroni correction. False negative results cannot be detected since we do not know the infinite population behind our predetermined study sample. By performing a post hoc power analysis including stroke severity (from NIHSS case-control calculations) we found rather modest statistical powers (between 5\% and 41\%), indicating a weak incentive for replicative studies to find an association between the selected SNPs and stroke severity (and even outcome, defined by the mRS nomenclature).

\section{Conclusion}

In this first attempt to study if stroke repair mechanisms linked to certain regions within the APOD and SIGMAR1 genes may also affect recovery from stroke and severity of stroke, we performed a candidate gene study including twelve SNPs from these two genetic regions.

Our data suggest that the rs7659 SNP within the $A P O D$ gene could be associated with risk for stroke and stroke severity at stroke onset. This mutation may decrease the levels of APOD and thereby diminish its protective cell signalling and antioxidant action. However, these associations showed only modest statistical significance, suggesting that our study may be underpowered despite the large sample size.

\section{Competing interests}

The authors report no conflict of interest.

\section{Authors' contributions}

Arne Lindgren and Håkan Lövkvist had the overall responsibility for this study, including research design, data analysis, results, discussion, and manuscript preparation. Arne Lindgren, Katarina Jood and Christina were involved in clinical samples and materials collection. Ann-Cathrin Jönsson contributed in data analysis. Holger Luthman selected the SNPs for analysis and discussed the results. Tadeusz Wieloch concieved the idea from experimental studies and discussed the results. All authors were involved in the research design, drafting the manuscript and have read and approved the final manuscript.

\section{Acknowledgements}

This study was supported by grants from the Swedish Research Council (K2008-65X-14605-06-03, K2011-65X-14605-09-6, K2010-61X-20378-04-3, 20112684, 2011-2652), the Swedish State (ALFGBG-148861), the Swedish Heart and Lung Foundation (20100256), the Yngve Land Foundation, the Crafoord Foundation, the King Gustaf V and Queen Victoria's Foundation, the Swedish Stroke Association, Lund University, Region Skåne, the EOS Freemason Foundation, the Tore Nilsson Foundation, the Swedish Brain Fund and the Lars Hierta Foundation. Lund University and the Sahlgrenska Academy are members of the International Stroke Genetics Consortium. Biobank services were provided by Region Skåne Competence Centre (RSKC Malmö), and Labmedicin Skåne, University and Regional Laboratories Region Skåne, Sweden. We thank Riksstroke for providing information on 3 month follow-up status for patients in Lund Stroke Register.

\section{Author details}

'Department of Clinical Sciences Lund, Neurology, Lund University, Lund, Sweden. ${ }^{2}$ Department of Neurology and Rehabilitation Medicine, Neurology, Skåne University Hospital, Lund, Sweden. ${ }^{3}$ Department of Health Sciences, Lund University, Lund, Sweden. ${ }^{4}$ Department of Clinical Sciences Malmö, Medical Genetics, Lund University, Malmö, Sweden. ${ }^{5}$ Department of Clinical Neuroscience and Rehabilitation, The Sahlgrenska Academy at University of Gothenburg, Institute of Neuroscience and Physiology, Gothenburg, Sweden. ${ }^{6}$ Department of Neurosurgery, Laboratory for Experimental Brain Research, Lund University, Lund, Sweden. 'R\&D Centre Skåne, Skåne University Hospital, SE-221 85 Lund, Sweden.

Received: 20 May 2014 Accepted: 23 September 2014

Published online: 28 September 2014

\section{References}

1. Luengo-Fernandez R, Paul NL, Gray AM, Pendlebury ST, Bull LM, Welch SJ, Cuthbertson FC, Rothwell PM: Population-based study of disability and institutionalization after transient ischemic attack and stroke: 10-year results of the Oxford Vascular Study. Stroke 2013, 44(10):2854-2861.

2. McNaughton $H$, Feigin $V$, Kerse $N$, Barber PA, Weatherall M, Bennett D, Carter K, Hackett M, Anderson C: Ethnicity and functional outcome after stroke. Stroke 2011, 42(4):960-964.

3. Feigin VL, Barker-Collo S, Parag V, Senior H, Lawes CM, Ratnasabapathy Y, Glen E: Auckland Stroke Outcomes Study. Part 1: Gender, stroke types, ethnicity, and functional outcomes 5 years poststroke. Neurology 2010, 75(18):1597-1607.

4. Rensink M, Schuurmans M, Lindeman E, Hafsteinsdottir T: Task-oriented training in rehabilitation after stroke: systematic review. J Adv Nurs 2009, 65(4):737-754.

5. Brogårdh C, Lexell J: Effects of cardiorespiratory fitness and muscle-resistance training after stroke. PM R 2012, 4(11):901-907.

6. Goldstein LB, Bushnell CD, Adams RJ, Appel LJ, Braun LT, Chaturvedi S, Creager MA, Culebras A, Eckel RH, Hart RG, Hinchey JA, Howard VJ, Jauch EC, Levine SR, Meschia JF, Moore WS, Nixon JV, Pearson TA: Guidelines for the primary prevention of stroke: a guideline for healthcare professionals from the American Heart Association/American Stroke Association. Stroke 2011 42(2):517-584.

7. Endres M, Engelhardt B, Koistinaho J, Lindvall O, Meairs S, Mohr JP, Planas A, Rothwell N, Schwaninger M, Schwab ME, Vivien D, Wieloch T, Dirnagl U: Improving outcome after stroke: overcoming the translational roadblock. Cerebrovasc Dis 2008, 25(3):268-278.

8. Pekna M, Pekny M: The neurobiology of brain injury. Cerebrum 2012, 2012:9 
9. Wieloch T, Nikolich K: Mechanisms of neural plasticity following brain injury. Curr Opin Neurobiol 2006, 16(3):258-264.

10. Cramer SC, Procaccio V: Correlation between genetic polymorphisms and stroke recovery: analysis of the GAIN Americas and GAIN International Studies. Eur J Neurol 2012, 19(5):718-724.

11. Lövkvist H, Smith JG, Luthman H, Höglund P, Norrving B, Kristoffersson U, Jönsson AC, Lindgren AG: Ischaemic stroke in hypertensive patients is associated with variations in the PDE4D genome region. Eur J Hum Genet 2008, 16(9):1117-1125.

12. Smith JG, Melander O, Lövkvist H, Hedblad B, Engström G, Nilsson P, Carlson J, Berglund $G$, Norrving $B$, Lindgren $A$ : Common genetic variants on chromosome 9p21 confers risk of ischemic stroke: a large-scale genetic association study. Circ Cardiovasc Genet 2009, 2(2):159-164.

13. Williams FM, Carter AM, Hysi AM, Surdulescu G, Hodgkiss D, Soranzo N, Traylor M, Bevan S, Dichgans M, Rothwell PM: Ischemic stroke is associated with ABO locus: The Euroclot study. Ann Neurol 2012, 73(1):16-31.

14. Bellenguez C, Bevan S, Gschwendtner A, Spencer CC, Burgess Al, Pirinen M, Jackson CA, Traylor M, Strange A, Su Z, Band G, Syme PD, Malik R, Pera J, Norrving B, Lemmens R, Freeman C, Schanz R, James T, Poole D, Murphy L, Segal H, Cortellini L, Cheng YC, Woo D, Nalls MA, Müller-Myhsok B, Meisinger C, Seedorf $U$, Ross-Adams $H$, et al: Genome-wide association study identifies a variant in HDAC9 associated with large vessel ischemic stroke. Nat Genet 2012, 44(3):328-333.

15. Gschwendtner A, Bevan S, Cole JW, Plourde A, Matarin M, Ross-Adams H, Meitinger T, Wichmann E, Mitchell BD, Furie K, Slowik A, Rich SS, Syme PD, MacLeod MJ, Meschia JF, Rosand J, Kittner SJ, Markus HS, Müller-Myhsok B, Dichgans M: Sequence variants on chromosome 9p21.3 confer risk for atherosclerotic stroke. Ann Neurol 2009, 65(5):531-539.

16. Holliday EG, Maguire JM, Evans TJ, Koblar SA, Jannes J, Sturm JW, Hankey GJ, Baker R, Golledge J, Parsons MW, Malik R, McEvoy M, Biros E, Lewis MD, Lincz LF, Peel R, Oldmeadow C, Smith W, Moscato P, Barlera S, Bevan S, Bis JC, Boerwinkle E, Boncoraglio GB, Brott TG, Brown RD Jr, Cheng YC, Cole JW, Cotlarciuc I, Devan WJ, et al: Common variants at 6p21.1 are associated with large artery atherosclerotic stroke. Nat Genet 2012, 44(10):1147-1151.

17. Traylor M, Farrall M, Holliday EG, Sudlow C, Hopewell JC, Cheng YC, Fornage M, Ikram MA, Malik R, Bevan S, Thorsteinsdottir U, Nalls MA, Longstreth W, Wiggins KL, Yadav S, Parati EA, Destefano AL, Worrall BB, Kittner SJ, Khan MS, Reiner AP, Helgadottir A, Achterberg S, Fernandez-Cadenas I, Abboud S, Schmidt R, Walters M, Chen WM, Ringelstein EB, O'Donnell M, et al: Genetic risk factors for ischaemic stroke and its subtypes (the METASTROKE collaboration): a meta-analysis of genome-wide association studies. Lancet Neurol 2012, 11(11):951-962.

18. Gretarsdottir S, Thorleifsson G, Manolescu A, Styrkarsdottir U, Helgadottir A, Gschwendtner A, Kostulas K, Kuhlenbäumer G, Bevan S, Jonsdottir T, Bjarnason H, Saemundsdottir J, Palsson S, Arnar DO, Holm H, Thorgeirsson G, Valdimarsson EM, Sveinbjörnsdottir S, Gieger C, Berger K, Wichmann HE, Hillert J, Markus H, Gulcher JR, Ringelstein EB, Kong A, Dichgans M, Gudbjartsson DF, Thorsteinsdottir U, Stefansson K: Risk variants for atrial fibrillation on chromosome $4 \mathrm{q} 25$ associate with ischemic stroke. Ann Neurol 2008, 64(4):402-409.

19. Gudbjartsson DF, Holm H, Gretarsdottir $S$, Thorleifsson G, Walters GB, Thorgeirsson G, Gulcher J, Mathiesen EB, Njølstad I, Nyrnes A, Wilsgaard T, Hald EM, Hveem K, Stoltenberg C, Kucera G, Stubblefield T, Carter S, Roden D, Ng MC, Baum L, So WY, Wong KS, Chan JC, Gieger C, Wichmann HE, Gschwendtner A, Dichgans M, Kuhlenbäumer G, Berger K, Ringelstein EB, et al: A sequence variant in ZFHX3 on $16 \mathrm{q} 22$ associates with atrial fibrillation and ischemic stroke. Nat Genet 2009, 41(8):876-878.

20. Biffi A, Anderson CD, Jagiella JM, Schmidt H, Kissela B, Hansen BM, JimenezConde J, Pires CR, Ayres AM, Schwab K, Cortellini L, Pera J, Urbanik A, Romero JM, Rost NS, Goldstein JN, Viswanathan A, Pichler A, Enzinger C, Rabionet R, Norving B, Tirschwell DL, Selim M, Brown DL, Silliman SL, Worrall BB, Meschia JF, Kidwell CS, Broderick JP, Greenberg SM, et al: APOE genotype and extent of bleeding and outcome in lobar intracerebral haemorrhage: a genetic association study. Lancet Neurol 2011, 10(8):702-709.

21. Maguire J, Thakkinstian A, Levi C, Lincz L, Bisset L, Sturm J, Scott R, Whyte S, Attia J: Impact of COX-2 rs5275 and rs20417 and GPIIla rs5918 polymorphisms on 90-day ischemic stroke functional outcome: a novel finding. J Stroke Cerebrovasc Dis 2011, 20(2):134-144.

22. Pearson-Fuhrhop KM, Minton B, Acevedo D, Shahbaba B, Cramer SC: Genetic variation in the human brain dopamine system influences motor learning and its modulation by L-Dopa. PLoS One 2013, 8(4):e61197.
23. Elliott DA, Weickert CS, Garner B: Apolipoproteins in the brain: implications for neurological and psychiatric disorders. Clin Lipidol 2010, 51(4):555-573.

24. Rassart E, Bedirian A, Do Carmo S, Guinard O, Sirois J, Terrisse L, Milne R: Apolipoprotein D. Biochim Biophys Acta 2000, 1482(1-2):185-198.

25. Ordonez C, Navarro A, Perez C, Martinez E, del Valle E, Tolivia J: Gender differences in apolipoprotein $D$ expression during aging and in Alzheimer disease. Neurobiol Aging 2012, 33(2):433 e411-420.

26. Thomas EA, Dean B, Pavey G, Sutcliffe JG: Increased CNS levels of apolipoprotein $D$ in schizophrenic and bipolar subjects: implications for the pathophysiology of psychiatric disorders. Proc Natl Acad Sci U S A 2001, 98(7):4066-4071.

27. Belloir B, Kovari E, Surini-Demiri M, Savioz A: Altered apolipoprotein D expression in the brain of patients with Alzheimer disease. J Neurosci Res 2001, 64(1):61-69.

28. Shibata N, Nagata T, Shinagawa S, Ohnuma T, Shimazaki H, Komatsu M, Kuerban B, Tomson K, Nakayama K, Yamada H, Arai H: Genetic association between APOA1 and APOD polymorphisms and Alzheimer's disease in a Japanese population. J Neural Transm 2013, 120(11):1599-1603.

29. Ordonez C, Navarro A, Perez C, Astudillo A, Martinez E, Tolivia J: Apolipoprotein $\mathrm{D}$ expression in substantia nigra of Parkinson disease. Histol Histopathol 2006, 21(4):361-366.

30. Navarro A, Alonso A, Garrido P, Gonzalez C, Gonzalez Del Rey C, Ordonez C, Tolivia J: Increase in placental apolipoprotein $\mathrm{D}$ as an adaptation to human gestational diabetes. Placenta 2010, 31(1):25-31.

31. Rickhag M, Deierborg T, Patel S, Ruscher K, Wieloch T: Apolipoprotein D is elevated in oligodendrocytes in the peri-infarct region after experimental stroke: influence of enriched environment. J Cereb Blood Flow Metab 2008, 28(3):551-562

32. Franz $G$, Reindl $M$, Patel SC, Beer R, Unterrichter I, Berger $T$, Schmutzhard $E$ Poewe W, Kampfl A: Increased expression of apolipoprotein D following experimental traumatic brain injury. J Neurochem 1999, 73(4):1615-1625.

33. Chen Y, Jia L, Wei C, Wang F, Lv H, Jia J: Association between polymorphisms in the apolipoprotein $\mathrm{D}$ gene and sporadic Alzheimer's disease. Brain Res 2008, 1233:196-202.

34. Ganfornina MD, Do Carmo S, Lora JM, Torres-Schumann S, Vogel M, Allhorn M, Gonzalez C, Bastiani MJ, Rassart E, Sanchez D: Apolipoprotein D is involved in the mechanisms regulating protection from oxidative stress. Aging Cell 2008, 7(4):506-515.

35. Bhatia S, Knoch B, Wong J, Kim WS, Else PL, Oakley AJ, Garner B: Selective reduction of hydroperoxyeicosatetraenoic acids to their hydroxy derivatives by apolipoprotein $\mathrm{D}$ : implications for lipid antioxidant activity and Alzheimer's disease. Biochemical J 2012, 442(3):713-721.

36. Kourrich S, Su TP, Fujimoto M, Bonci A: The sigma-1 receptor: roles in neuronal plasticity and disease. Trends Neurosci 2012, 35(12):762-771.

37. Ruscher K, Shamloo M, Rickhag M, Ladunga I, Soriano L, Gisselsson L, Toresson H, Ruslim-Litrus L, Oksenberg D, Urfer R, Johansson BB, Nikolich $K$, Wieloch T: The sigma-1 receptor enhances brain plasticity and functional recovery after experimental stroke. Brain 2011, 134(3):732-746.

38. Kishi T, Yoshimura R, Okochi T, Fukuo Y, Kitajima T, Okumura T, Tsunoka T, Kawashima K, Yamanouchi Y, Kinoshita Y, Umene-Nakano W, Naitoh H, Nakamura J, Ozaki N, Iwata N: Association analysis of SIGMAR1 with major depressive disorder and SSRI response. Neuropharmacology 2010, 58(7):1168-1173.

39. Ishiguro H, Ohtsuki T, Toru M, Itokawa M, Aoki J, Shibuya H, Kurumaji A, Okubo Y, Iwawaki A, Ota K, Shimizu H, Hamaguchi H, Arinami T: Association between polymorphisms in the type 1 sigma receptor gene and schizophrenia. Neurosci Lett 1998, 257(1):45-48.

40. Miyatake R, Furukawa A, Matsushita S, Higuchi S, Suwaki H: Functional polymorphisms in the sigma1 receptor gene associated with alcoholism. Biol Psychiatry 2004, 55(1):85-90.

41. Feher A, Juhasz A, Laszlo A, Kalman J Jr, Pakaski M, Kalman J, Janka Z: Association between a variant of the sigma-1 receptor gene and Alzheimer's disease. Neurosci Lett 2012, 517(2):136-139.

42. Hallström B, Jönsson AC, Nerbrand C, Petersen B, Norrving B, Lindgren A: Lund Stroke Register: hospitalization pattern and yield of different screening methods for first-ever stroke. Acta Neurol Scand 2007, 115(1):49-54.

43. Jood K, Ladenvall C, Rosengren A, Blomstrand C, Jern C: Family history in ischemic stroke before 70 years of age: the Sahlgrenska Academy Study on Ischemic Stroke. Stroke 2005, 36(7):1383-1387.

44. Goldstein $L B$, Bertels $C$, Davis JN: Interrater reliability of the NIH stroke scale. Arch Neurol 1989, 46(6):660-662. 
45. Multicenter trial of hemodilution in ischemic stroke-background and study protocol. Scandinavian Stroke Study Group. Stroke 1985, 16(5):885-890

46. Gray LJ, Ali M, Lyden PD, Bath PM: Interconversion of the National Institutes of Health Stroke Scale and Scandinavian Stroke Scale in acute stroke. J Stroke Cerebrovasc Dis 2009, 18(6):466-468.

47. Adams HP Jr, Bendixen BH, Leira E, Chang KC, Davis PH, Woolson RF, Clarke WR, Hansen MD: Antithrombotic treatment of ischemic stroke among patients with occlusion or severe stenosis of the internal carotid artery: A report of the Trial of Org 10172 in Acute Stroke Treatment (TOAST). Neurology 1999, 53(1):122-125

48. Eriksson M, Appelros P, Norrving B, Terent A, Stegmayr B: Assessment of functional outcome in a national quality register for acute stroke: can simple self-reported items be transformed into the modified Rankin Scale? Stroke 2007, 38(4):1384-1386.

49. van Swieten JC, Koudstaal PJ, Visser MC, Schouten HJ, van Gijn J: Interobserver agreement for the assessment of handicap in stroke patients. Stroke 1988, 19(5):604-607.

50. Olsson S, Jood K, Blomstrand C, Jern C: Genetic variation on chromosome 9p21 shows association with the ischaemic stroke subtype large-vessel disease in a Swedish sample aged $</=70$. Eur J Neurol 2011, 18(2):365-367.

51. Starby $H$, Delavaran $H$, Andsberg $G$, Lövkvist $H$, Norrving B, Lindgren $A$ : Multiplicity of risk factors in ischemic stroke patients: relations to age, sex, and subtype - a study of 2,505 patients from the Lund Stroke Register. Neuroepidemiology 2014, 42(3):161-168.

52. Flossmann E, Schulz UG, Rothwell PM: Systematic review of methods and results of studies of the genetic epidemiology of ischemic stroke. Stroke 2004, 35(1):212-227.

53. Schutze MP, Peterson PA, Jackson MR: An N-terminal double-arginine motif maintains type II membrane proteins in the endoplasmic reticulum. EMBO J 1994, 13(7):1696-1705.

54. Hansen T, Hemmingsen RP, Wang AG, Olsen L, Timm S, Soeby K, Jakobsen KD, Fenger M, Parnas J, Rasmussen HB, Werge T: Apolipoprotein D is associated with long-term outcome in patients with schizophrenia. Pharmacogenomics J 2006, 6(2):120-125.

doi:10.1186/s12883-014-0191-2

Cite this article as: Lövkvist et al:: Variations in apolipoprotein D and sigma non-opioid intracellular receptor 1 genes with relation to risk, severity and outcome of ischemic stroke. BMC Neurology 2014 14:191.

\section{Submit your next manuscript to BioMed Central and take full advantage of:}

- Convenient online submission

- Thorough peer review

- No space constraints or color figure charges

- Immediate publication on acceptance

- Inclusion in PubMed, CAS, Scopus and Google Scholar

- Research which is freely available for redistribution 